\title{
O valor atribuído à saúde bucal: um estudo com acadêmicos iniciantes de quatro cursos de graduação
}

\author{
The value attributed to oral health: \\ a study with undergraduate students of four courses \\ El valor atribuido a la salud bucal: \\ un estudio con estudiantes de pregrado de cuatro cursos \\ José Klidenberg de OLIVEIRA-JÚNIOR ${ }^{1}$ \\ Luan Éverton Galdino BARNABÉ ${ }^{2}$ \\ Marisley Layrtha SANTOS ${ }^{3}$ \\ Alynne MACEDO ${ }^{4}$ \\ Rachel Queiroz Ferreira RODRIGUES ${ }^{5}$ \\ Maria Carolina Bandeira MACENA ${ }^{5}$
}

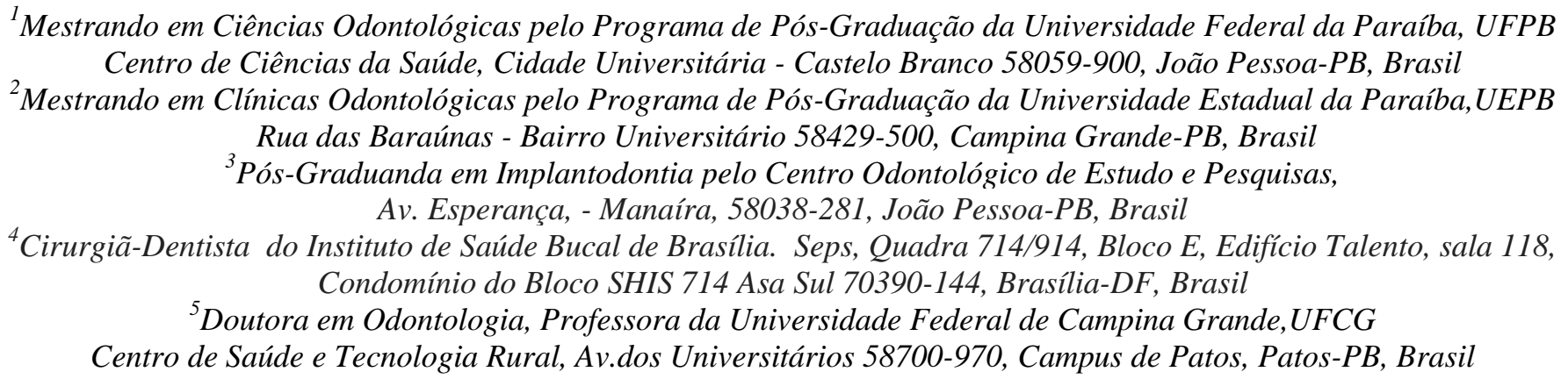

\section{Resumo}

A prática da educação em saúde permite promover no indivíduo alterações positiva frente aos hábitos de higiene bucal, estimulandoos a aprender e capacitando-os a tomar decisões adequadas quanto à sua saúde bucal. O presente estudo teve como objetivo avaliar o nível de conhecimento em saúde bucal de estudantes universitários ao iniciar o curso superior. $\mathrm{O}$ estudo procedeu-se com alunos regularmente matriculados no segundo semestre 2015.2 de acordo com a listagem repassada pela coordenação de cada curso pertencente ao Campus de Patos-PB da Universidade Federal de Campina Grande/Paraíba (UFCG). Foram obtidos 128 formulários $(\mathrm{n}=128)$ referentes aos quatro cursos analisados. Os dados foram coletados e analisados por meio da estatística descritiva, utilizando software Statistical Package for the Social Sciences (SPSS) versão 22.0. A maioria dos estudantes permanece na instituição de forma integral (Odontologia 61,3\%; Ciências Biológicas 79,5\%; Medicina Veterinária 60,6\% e Engenharia Florestal 60\%) e a forma de higienização utilizada durante esse período foi à escovação, o número de acadêmicos que nunca participou de uma atividade de saúde bucal é considerável (Odontologia 51,7\%; Ciências Biológicas 51,3\%; Medicina Veterinária 60,6\% e Engenharia Florestal 48\%), e consideram sua saúde bucal boa (Odontologia 74,1\%; Ciências Biológicas 69,2\%; Medicina Veterinária 69,6\% e Engenharia Florestal $60 \%$ ). De acordo com as respostas obtidas, a amostra do referido estudo apresenta um conhecimento razoável sobre saúde bucal necessitando de medidas preventivas/interceptativas no âmbito da saúde bucal.

Descritores: Educação em Saúde Bucal; Estudantes; Saúde Pública.

\begin{abstract}
The practice of health education allows promoting in the individuals positive changes in oral hygiene habits, stimulating them to learn and enabling them to make appropriate decisions regarding their oral health. The aim of this study was to evaluate the level of oral health knowledge of university students when they started higher education. The study was carried out with students enrolled in the second semester of 2015.2 According to the list provided by the coordination of each course belonging to the Patos-PB Campus of the Federal University of Campina Grande / Paraíba (UFCG), 128 forms were obtained $(n=128)$ referring to the four courses analyzed. data were collected and analyzed using descriptive statistics using Statistical Package for Social Sciences (SPSS) software version 22.0. The majority of the students stays in the institution (Dentistry 61.3\%, Biological Sciences 79.5\%, Veterinary Medicine $60.6 \%$ and Forest Engineering 60\%) and the form of hygiene used during this period was brushing (51.7\%, Biological Sciences 51.3\%, Veterinary Medicine $60.6 \%$ and Forestry Engineering 48\%) and they consider their oral health as good (Dentistry 74,1\%; Biological Sciences 69.2\%, Veterinary Medicine 69.6\% and Forest Engineering 60\%). According to the answers obtained, the sample of the mentioned study presents a regular oral health necessitating preventive/ interceptive measures in the field of oral health.

Descriptors: Health Education, Dental; Students; Public Health.
\end{abstract}

\section{Resumen}

La práctica de educación sanitaria permite al individuo para promover modificaciones a plazo positivos en los hábitos de higiene oral, animándoles a aprender y capacitando para tomar decisiones apropiadas sobre su salud oral. Este estudio tuvo como objetivo evaluar el nivel de conocimiento sobre la salud oral de los estudiantes universitarios para iniciar la universidad. El estudio llevado a cabo con los estudiantes matriculados en el segundo semestre 2015.2 según la lista aprobada para la coordinación de cada curso perteneciente al Campus de Patos-PB en la Universidad Federal de Campina Grande / Paraíba (UFCG) se obtuvieron 128 formas ( $\mathrm{n}=128)$ para los cuatro cursos analizados. Los datos fueron recogidos y analizados utilizando estadística descriptiva, utilizando el software Paquete Estadístico para Ciencias Sociales (SPSS) versión 22.0. La mayoría de los estudiantes permanecen en la institución de forma completa (Odontología 61,3\%; Ciencias Biológicas79,5\%, Medicina Veterinaria 60,6\% y Ingeniería Forestal 60\%) y la forma de la higiene utilizado durante este período fue el cepillado, el número de académicos que nunca han participado en una actividad de la salud oral es considerable (Odontología 51,7\%; 51,3\% Ciencias Biológicas, Medicina Veterinaria 60,6\% y Ingeniería Forestal 48\%), y considerar su buena salud oral (Odontología 74,1\%, Ciencias Biológicas 69,2\%, Medina Veterinária 69,6\% y Ingeniería Forestal 60\%). De acuerdo con las respuestas, la muestra del estudio presenta un conocimiento razonable de las necesidades de salud oral de medidas preventivas / de intercepción en la salud oral.

Descriptores: Educación en Salud Dental; Estudiantes; Salud Pública.

\section{INTRODUÇÃO}

No Brasil, o quadro epidemiológico das doenças bucais ainda apresenta níveis de precariedade. A educação e motivação são fatores capazes de desenvolver o interesse pela manutenção da condição de saúde, despertando nos indivíduos consciência das reais causas de seus problemas ${ }^{1,2}$. As ações de educação em saúde são essenciais à melhoria da 
qualidade de vida, uma vez que a prevenção e promoção da saúde são a base para o desenvolvimento social e pessoal do indivíduo ${ }^{3}$.

A prática da educação em saúde permite promover no indivíduo alterações positivas frente aos hábitos de higiene bucal, estimulando-os a aprender e capacitando-os a tomar decisões adequadas quanto à sua saúde bucal ${ }^{4}$.

As estratégias inseridas nos programas voltados à educação em saúde estimulam ao indivíduo a atingir um nível de higiene bucal e um patamar de condição periodontal que o mantenha com uma dentição funcional e com uma estética favorável por toda a vida ${ }^{5}$.

Em função das desigualdades sociais e da má distribuição de renda, uma mesma camada da sociedade tende a beneficiar-se dos serviços odontológicos. Contudo, o nível de conhecimento a respeito do processo de determinação da doença, assim como a incorporação de políticas de saúde bucais coletivas, é capaz de produzir efeitos em todas as camadas sociais ${ }^{6,7}$.

A saúde bucal das populações não resulta apenas da prática clínica rotineira, mas de construções sociais operadas de modo consciente em cada situação concreta, incluindo os profissionais de saúde, dentre eles, os cirurgiões-dentistas ${ }^{8}$. $\mathrm{Na}$ atualidade o nível de conhecimento sobre saúde bucal dos indivíduos vem aumentando consequentemente refletindo em uma melhor qualidade de vida ${ }^{9}$.

Partindo desse pressuposto, analisar o valor que os indivíduos atribuem à saúde bucal tem sido uma constante nas pesquisas desenvolvidas no campo de Educação em Saúde envolvendo a Odontologia, pois os indivíduos costumam não procurar o atendimento odontológico justamente pela não percepção de suas necessidades ${ }^{10}$.

No caso de acadêmicos iniciantes, torna-se importante investigar o valor que esse grupo atribui a saúde bucal, justamente pelo fato desses estudantes comporem um grupo que está em fase de transição na sua vida, pois é sabido que o nível de responsabilidade após o ingresso na academia aumenta. Acrescenta-se a isso o fato do estudante na maioria das vezes, morar sozinho e distante da família, estudando em tempo integral, o que consome grande parte do seu tempo, podendo esses fatores refletir na qualidade da saúde geral e/ou bucal do indivíduo ${ }^{11,12}$

Com base nisso, o propósito desse estudo é avaliar o nível de conhecimento em saúde bucal de estudantes universitários ao iniciar o curso superior, de modo a fornecer subsídios para futuras estratégias preventivas e educativas no âmbito da saúde bucal da referida instituição pesquisada.

\section{MATERIAL E MÉTODO}

Realizou-se um estudo observacional, como método indutivo e procedimento comparativo, estatístico-descritivo, através da técnica de documentação direta extensiva através do formulário ${ }^{13}$.

O cenário de coleta de dados foi constituído por salas de aula do Centro de Saúde e Tecnologia Rural da Universidade Federal de Campina Grande Patos-PB, após o parecer favorável do Comitê de Ética e Pesquisa do Hospital Universitário Alcides Carneiro vinculado à referida instituição (Protocolo ${ }^{\circ}$ 895.655).

$\mathrm{O}$ estudo procedeu-se com alunos regularmente matriculados no segundo semestre 2015.2 de acordo com a listagem repassada pela coordenação de cada curso pertencente ao Campus de Patos-PB da Universidade Federal de Campina Grande (UFCG). Os cursos pesquisados foram os seguintes: Ciências Biológicas, Medicina Veterinária, Odontologia e Engenharia Florestal. O estudo foi desenvolvido entre os meses de julho a dezembro de 2015.

Previamente à aplicação do formulário foi realizada um estudo piloto com 10 alunos $(n=10)$ com a finalidade de verificar se $\mathrm{o}$ formulário apresentava fidedignidade (qualquer pessoa que o aplique obterá sempre os mesmos resultados), validade (os dados recolhidos são necessários à pesquisa) e operatividade (vocabulário acessível e significado claro). O estudo piloto não indicou prováveis alterações no instrumento da pesquisa.

Em seguida procedeu-se a aplicação dos formulários por uma pessoa treinada a fim de suprir supostas dúvidas sem influenciar nas respostas. Foram obtidos 128 formulários $(\mathrm{n}=128)$ referentes aos alunos dos quatro cursos analisados, dentre as variáveis verificadas encontram-se dados sociais (sexo e faixa etária) e questões relativas à saúde bucal (visitas ao dentista, escovação, uso do fio dental entre outras).

Os estudantes foram abordados em sala de aula da referida instituição, sendo o critério de inclusão adotado na pesquisa baseado na presença dos mesmos no dia da aplicação do formulário, na concordância em participar e ter idade superior a 18 anos.

Os dados foram analisados através do software Statistical Package for the Social Sciences (SPSS) versão 22.0 .

\section{RESULTADOS}

Foram avaliados 128 alunos por meio dos questionários, com maior prevalência para o sexo feminino nos quatros cursos analisado e com idade média de 19 anos (Tabela 1).

Tabela 1. Distribuição da frequência relacionada ao sexo e idade dos acadêmicos iniciantes dos quatros cursos analisados

\begin{tabular}{ccc|c}
\hline \multirow{3}{*}{ CURSOS } & \multicolumn{2}{c|}{ SEXO (fi) } & IDADE \\
& MASCULINO & FEMININO & \\
\hline ODONTOLOGIA & $7,03 \%$ & $17,20 \%$ & 19,5 \\
CIÊNCIAS BIOLÓGICAS & $10,15 \%$ & $20,33 \%$ & 19,5 \\
MEDICINA VETERINÁRIA & $11,71 \%$ & $14,06 \%$ & 18,6 \\
ENGENHARIA & $7,81 \%$ & $11,71 \%$ & 18,9 \\
FLORESTAL & & & \\
TOTAL & $\mathbf{3 6 , 7 \%}$ & $\mathbf{6 3 , 3} \%$ & $\mathbf{1 9 , 1}$ \\
\hline
\end{tabular}

fi: frequência absoluta $\bar{x}$ : média

Quando indagados à permanência de forma integral na universidade, exceto o curso de Ciências Biológicas, a resposta com maior frequência, foi "Frequentemente", mostrando que a maioria dos acadêmicos permanece mais de um turno na instituição (Tabela 2).

No entanto quando aqueles que afirmaram permanecer na faculdade foram questionados sobre higienização nesse período, observou-se que $12 \%$ dos alunos do curso de Medicina Veterinária não realizavam qualquer forma de higienização, bem como $32,3 \%$ dos alunos da Odontologia, 33,3\% dos acadêmicos de Engenharia Florestal e a maioria dos alunos de Ciências Biológicas $(66,7 \%)$, obtendo-se assim o maior índice nesse questionamento. No entanto, boa parte dos alunos afirma realizar alguma forma de higienização, utilizando escova e creme dental como método de limpeza (Tabela 2).

Em relação à frequência da higienização bucal, o número de três escovações diárias obteve maior prevalência 
sobre outras quantidades de escovações diárias entre os quatros cursos, entretanto, a porcentagem dos alunos de Odontologia $(93,5 \%)$ destaca-se sobre os demais cursos. Também foi questionada a frequência no uso do fio dental e assim como a escovação, obteve-se uma maior prevalência na resposta padrão, "Uma vez ao dia", em todos os períodos com exceção do curso de Medicina Veterinária, que utiliza o fio dental "Duas vezes ao dia". Quando questionados sobre ir ao dentista nos últimos 12 meses houve uma unanimidade entre os cursos com a resposta positiva "Sim", sendo a maior prevalência novamente no curso de Odontologia com 90,3\% dos alunos (Tabela 3).

Tabela 2. Frequência absoluta do tempo de permanência na universidade e método de higienização quando o acadêmico permanece na instituição

\begin{tabular}{|c|c|c|c|c|}
\hline CURSOS & $\begin{array}{c}\text { Odontologia } \\
\text { fi }(\%)\end{array}$ & $\begin{array}{c}\text { Ciências } \\
\text { Biológicas } \\
\text { fi (\%) }\end{array}$ & $\begin{array}{l}\text { Medicina } \\
\text { Veterinária } \\
\text { fi }(\%)\end{array}$ & $\begin{array}{l}\text { Engenharia } \\
\text { Florestal } \\
f i(\%)\end{array}$ \\
\hline \multirow{2}{*}{$\begin{array}{l}\text { Período } \\
\text { integral na } \\
\text { faculdade }\end{array}$} & $\begin{array}{l}\text { Frequentemente } \\
61,3 \%\end{array}$ & $\begin{array}{l}\text { Frequentemente } \\
79,5 \%\end{array}$ & $\begin{array}{l}\text { Frequentemente } \\
60,6 \%\end{array}$ & $\begin{array}{c}\text { Frequentemente } \\
60 \%\end{array}$ \\
\hline & $\begin{array}{c}\text { Às vezes } \\
38,7 \%\end{array}$ & $\begin{array}{l}\text { Às vezes } \\
20,5 \%\end{array}$ & $\begin{array}{l}\text { Às vezes } \\
39,4 \%\end{array}$ & $\begin{array}{l}\text { Às vezes } \\
40 \%\end{array}$ \\
\hline \multirow{5}{*}{$\begin{array}{l}\text { Higienização } \\
\text { quando } \\
\text { permanece } \\
\text { na } \\
\text { universidade }\end{array}$} & $\begin{array}{c}\text { Escova + creme } \\
\text { dental + fio } \\
3,2 \%\end{array}$ & $\begin{array}{c}\text { Escova + creme } \\
\text { dental + fio } \\
7,7 \%\end{array}$ & $\begin{array}{c}\text { Escova + creme } \\
\text { dental + fio } \\
12 \%\end{array}$ & $\begin{array}{c}\text { Escova + creme } \\
\text { dental + fio } \\
15,2 \%\end{array}$ \\
\hline & $\begin{array}{c}\text { Escova }+ \text { creme } \\
\text { dental } \\
51,6 \%\end{array}$ & $\begin{array}{c}\text { Escova + creme } \\
\text { dental } \\
20,5 \%\end{array}$ & $\begin{array}{c}\text { Escova }+ \text { creme } \\
\text { dental } \\
60 \%\end{array}$ & $\begin{array}{c}\text { Escova + creme } \\
\text { dental } \\
21,2 \%\end{array}$ \\
\hline & Fio $12,9 \%$ & Fio $5,1 \%$ & Fio $4 \%$ & Fio $9,1 \%$ \\
\hline & Não faço $32,3 \%$ & Não faço 66,7\% & Palito $12 \%$ & Palito $21,2 \%$ \\
\hline & & & Não faço 12\% & Não faço 33,3\% \\
\hline
\end{tabular}

Tabela 3. Distribuição da frequência absoluta dos itens relacionados aos cuidados e autoavaliação da saúde bucal dos acadêmicos iniciantes

\begin{tabular}{|c|c|c|c|c|}
\hline CURSOS & $\begin{array}{l}\text { Odontologia } \\
f i(\%)\end{array}$ & $\begin{array}{c}\text { Ciências } \\
\text { Biológicas } \\
\text { fi }(\%)\end{array}$ & $\begin{array}{c}\text { Medicina } \\
\text { Veterinária } \\
\quad f i(\%)\end{array}$ & $\begin{array}{l}\text { Engenharia } \\
\text { Florestal } \\
\text { fi }(\%)\end{array}$ \\
\hline $\begin{array}{l}\text { Frequência } \\
\text { na escovação }\end{array}$ & $\begin{array}{c}2 \text { vezes ao } \\
\text { dia } \\
6,5 \% \\
3 \text { vezes ao } \\
\text { dia } \\
93,5 \%\end{array}$ & $\begin{array}{c}2 \text { vezes ao dia } \\
15,4 \% \\
3 \text { vezes ao dia } \\
53,8 \% \\
\text { Mais de } 3 \\
\text { vezes ao dia } \\
30,8 \%\end{array}$ & $\begin{array}{c}1 \text { vez ao dia } 6,1 \% \\
2 \text { vezes ao dia } \\
9,1 \% \\
3 \text { vezes ao dia } \\
54,5 \% \\
\text { Mais de } 3 \text { vezes ao } \\
\text { dia } 30,3 \% \\
\end{array}$ & $\begin{array}{c}2 \text { vezes ao dia } \\
8 \% \\
3 \text { vezes ao dia } \\
1560 \% \\
\text { Mais de três } \\
\text { vezes ao dia } \\
32 \%\end{array}$ \\
\hline $\begin{array}{l}\text { Frequência } \\
\text { no uso de fio } \\
\text { dental }\end{array}$ & $\begin{array}{c}1 \text { vez ao dia } \\
51,6 \% \\
2 \text { vezes ao } \\
\text { dia } \\
32,3 \% \\
\\
3 \text { vezes ao } \\
\text { dia } \\
6,5 \% \\
\text { Nunca } \\
9,7 \%\end{array}$ & $\begin{array}{c}1 \text { vez ao dia } \\
35,9 \% \\
2 \text { vezes ao dia } \\
33,3 \% \\
3 \text { vezes ao dia } \\
15,4 \% \\
\text { Mais de três } \\
\text { vezes ao dia } \\
2,6 \% \\
\text { Nunca } 12,8 \%\end{array}$ & $\begin{array}{c}1 \text { vez ao dia } \\
27,3 \% \\
2 \text { vezes ao dia } \\
36,4 \% \\
3 \text { vezes ao dia } \\
12,1 \% \\
\text { Nunca } 24,2 \%\end{array}$ & $\begin{array}{c}1 \text { vez ao dia } \\
36 \% \\
2 \text { vezes ao dia } \\
16 \% \\
3 \text { vezes ao dia } \\
12 \% \\
\text { Nunca } 32,0 \%\end{array}$ \\
\hline $\begin{array}{c}\text { Foi ao } \\
\text { dentista nos } \\
\text { últimos } 12 \\
\text { meses }\end{array}$ & $\begin{array}{c}\quad \operatorname{Sim} \\
28(90,3 \%)\end{array}$ & $\begin{array}{c}\operatorname{Sim}_{30}(76,9 \%) \\
\text { (7) }\end{array}$ & $\begin{array}{c}\text { Sim } \\
27(81,8 \%)\end{array}$ & $\begin{array}{c}\operatorname{Sim}_{19}(76 \%) \\
\text { (1) }\end{array}$ \\
\hline $\begin{array}{l}\text { Você tem } \\
\text { cárie? }\end{array}$ & $\begin{array}{c}\text { Não } \\
27(87 \%)\end{array}$ & $\begin{array}{c}\text { Não } \\
18(46,1 \%)\end{array}$ & $\begin{array}{c}\text { Não } \\
20(60,6 \%)\end{array}$ & $\begin{array}{c}\text { Não } \\
13(52 \%)\end{array}$ \\
\hline $\begin{array}{l}\text { Avaliação da } \\
\text { saúde bucal }\end{array}$ & $\begin{array}{c}\text { Boa } \\
23(74,1 \%)\end{array}$ & $\begin{aligned} & \text { Boa } \\
27 & (69,2 \%)\end{aligned}$ & $\begin{array}{c}\text { Boa } \\
23(69,6 \%)\end{array}$ & $\begin{array}{c}\text { Boa } \\
15(60 \%)\end{array}$ \\
\hline $\begin{array}{l}\text { Participou de } \\
\text { palestra } \\
\text { educativa }\end{array}$ & $\begin{array}{l}\text { Sim - 48,3\% } \\
\text { Não - 51,7\% }\end{array}$ & $\begin{array}{l}\text { Sim - 48,7\% } \\
\text { Não - } 51,3 \%\end{array}$ & $\begin{array}{l}\text { Sim }-42,4 \% \\
\text { Não - } 57,6 \%\end{array}$ & $\begin{array}{l}\text { Sim - } 52 \% \\
\text { Não- } 48 \%\end{array}$ \\
\hline
\end{tabular}

As questões seguintes dos formulários avaliavam a autopercepção dos alunos sobre presença de cárie e autoavaliação da saúde bucal. No que diz respeito à presença de cárie, a maioria dos alunos afirmou "Não ter", sendo que a maior parte dos alunos de Ciências Biológicas afirmava "Não saber". Na autoavaliação da saúde bucal obteve-se uma resposta unânime de "Boa" com maior prevalência entre os alunos do curso de Odontologia $(74,1 \%)$. Sobre a participação em palestras educativas, grande parte dos acadêmicos nunca participou de uma atividade educativa em saúde bucal (Tabela 3).

\section{DISCUSSÃO}

Analisando os dados obtidos percebe-se que nem todos acadêmicos que permanecem integralmente realizam a higienização pelo método mais simples, que é a escovação. A escovação é o método eficiente no combate à placa dentária e a doença gengival. Fato esse documentado em estudos clássicos na literatura, onde a falta de escovação dental resultou em um rápido acúmulo de biofilme dental e consequentemente desenvolvimento da doença gengival por um período de três semanas ${ }^{14}$.

A maioria dos estudantes realiza esse procedimento "três vezes ao dia", esses dados corroboram com estudos encontrados na literatura ${ }^{15,16}$, em que dentre os questionamentos avaliados na pesquisa estava a frequência de escovação, e os resultados demonstraram que $45 \%$ da amostra realizava o procedimento três vezes ao dia, outros $23,1 \%$ e $25,9 \%$ realizavam mais de "três" e "duas vezes", respectivamente.

Ainda sobre os métodos de higienização, o fio dental constitui juntamente com a escovação os principais meios mecânicos, devendo ser empregado nos espaços interdentários, locais em que a escovação não é efetiva na remoção dos resíduos de biofilme dental. Analisando a frequência da utilização dos graduandos nesse estudo, percebe-se que nos cursos de Odontologia, Ciências Biológicas e Engenharia Florestal os estudantes só utilizam esse meio de higienização "uma vez ao dia", fato que corrobora com os achados literários ${ }^{9}$, onde um total de $34,2 \%$ dos estudantes relatou utilizar o fio dental uma vez ao dia, $36,0 \%$ duas vezes ao dia e apenas $29,8 \%$ três vezes ou mais.

No mesmo estudo ${ }^{9}$ avaliou-se a frequência de visitas ao dentista nos últimos 12 meses, $45,5 \%$ visitam anualmente o dentista, resultados semelhantes ao estudo em questão, em que mais de $50 \%$ dos acadêmicos dos quatros cursos também procuram os consultórios odontológicos nesse mesmo período de tempo.

A cárie dentária é uma doença de origem multifatorial e complexa que inclui microbiota, dieta, hospedeiro, além de fatores coadjuvantes como socioeconômicos e ambientais, atinge diversas faixas etárias, e é a patologia mais comum da cavidade bucal ${ }^{17}$. Como nesse estudo o principal foco é avaliar a condição de saúde bucal dos estudantes envolvidos, fez-se necessário indagá-los sobre a presença da cárie dentária. Nos cursos estudados, mais da metade dos alunos entrevistados afirmam não possuir cárie, enquanto que $54 \%$ do curso de Ciências Biológicas não sabem se tem a doença, esses dados são preocupantes, pois existe uma parcela de alunos que falam não saber ou já possui a doença cárie, sendo necessárias medidas interceptativas, pois entende que a progressão da doença cárie pode acarretar a perda do elemento dentário, ocasionando problemas não só estéticos como também oclusais ${ }^{17}$.

A odontologia contemporânea está voltada para a promoção e prevenção de saúde bucal, visando à diminuição dos índices de saúde bucal ${ }^{18}$. Este fato não poderia ser diferente, uma vez que, com a evolução dessa área no campo científico, proporcionou uma bagagem científica suficiente 
para permitir que as doenças mais prevalentes da cavidade bucal (cárie e doença periodontal) sejam evitadas ${ }^{18,19}$.

$\mathrm{O}$ desenvolvimento das atividades em educação em saúde para a população, uma vez que desempenha papel relevante na prevenção dos problemas bucais, pois a educação assume destaque na obtenção de uma boa saúde bucal, favorecendo o desenvolvimento do pensamento crítico sobre as causas dos seus problemas de saúde bucal, despertando o interesse em cuidar da sua saúde ${ }^{20}$.

Apesar disso, a população estudada mostrou-se com um déficit considerável no que diz respeito à participação em atividades educativas. O curso de Medicina Veterinária seguido do Curso de Odontologia $(42,4 \%$ e $48,3 \%$ respectivamente) caracterizou-se pelos menores índices na participação de tais atividades. Esse percentual é bastante representativo em um país em que, há quase 20 anos, existe uma Constituição que determina o dever de garantir ações integrais em saúde. Infelizmente esses dados não estão isolados apenas nesse estudo; outros trabalhos mostraram o acesso deficiente de informações relacionadas a saúde bucal, como em um estudo com 93 idosos em que apenas 32 idosos $(16,4 \%)$ disseram ter participado de atividades educativas na área de saúde bucal em toda sua vida ${ }^{21}$.

\section{CONCLUSÃO}

Diante do exposto, concluiu-se que a população estudada apresentou um conhecimento razoável em relação aos cuidados com a saúde bucal. Os dados indicam a necessidade de implantar medidas preventivo-educativas com intuito subsidiar informações sobre saúde bucal, tendo em vista que grande parcela dos estudantes nunca teve acesso a palestras educativas sobre o assunto.

\section{REFERÊNCIAS}

1. Ribas MO, Orellana B, Fronza F, Gasparim GF, Mello GS, Simas MLS Neta, et al. Estudo epidemiológico das maloclusões em escolares de 6 a 8 anos na cidade de Curitiba - Paraná. Rev Sul-Bras Odontol. 2004; 1(1):229.

2. Kruschewsky JE, Kruschewsky ME, Cardoso JP. Experiências pedagógicas e a educação popular em saúde: A pedagogia tradicional versus a problematizadora. Rev Saúde Com. 2008;4(2):160-76.

3. Eskenazi EMS, Sousa KG, Agostini LTP, Barbosa TS, Castelo PM. Avaliação da experiência de cárie e qualidade de vida relacionada à saúde bucal de escolares. Rev Bras Promoç. Saúde. 2015; 28(2):198205.

4. Oliveira MF, Zanchett S, Berndt RLE, Moraes MVM. Motivação no controle do biofilme dental e o aprendizado em relação à saúde bucal em escolares. Publ UEPG Biol Health Sci. 2012; 18(2):115-20.

5. Ditterich RG, Porteto PP, Wambier DS, Pilatti GL, Santos FA. Higiene bucal e motivação no controle do biofilme dental. Odontol Clín-Cient. 2007; 6(2):123-8.

6. Silva GG. Estudo qualitativo sobre educação em saúde bucal [dissertação]. Florianópolis: Universidade Federal de Santa Catarina; 2012.

7. Petry PC, Victora CG, Santos IS. Adultos livres de cárie: estudo de casos e controles sobre conhecimentos, atitudes e práticas preventivas. Cad Saúde Pública. 2000; 16(1):145-53.

8. Narvai PC. Saúde bucal coletiva: caminhos da odontologia sanitária à bucalidade. Rev Saúde Pública. 2006; 40(N Esp):141-7.
9. Menezes RD, Cavalcanti AL. Estudo comparativo da informação em saúde bucal entre estudantes de cursos da área da saúde. Pesq Bras Odontoped Clin Integr. 2003; $3(2): 27-33$.

10. Silva SRC, Fernandes RAC. Autopercepção das condições de saúde bucal por idosos. Rev Saúde. Pública. 2001; 35(4):349-55.

11. Castro FC. Os temores na formação e prática da medicina: aspectos pscicológicos. Rev bras educ méd. 2004; 28(1):38-45.

12. Morrison J, Moffat K. More on medical student stress. Med Educ. 2001, 35(7):617-8.

13. Lakatos EM, Marconi MA. Fundamentos da metodologia científica. $5^{\circ}$ ed. São Paulo: Atlas; 2003.

14. Toassi, RFC, Petry, PC. Motivação no controle do biofilme dental e sangramento gengival em escolares. Rev Saúde Pública. 2002; 36(5):634-7.

15. Olympio KPK, Bardal PAP, Henriques JFC, Bastos JRDM. Prevenção de cárie dentária e doença periodontal em Ortodontia: uma necessidade imprescindível. $\mathrm{R}$ Dental Press Ortodon Ortop Facial. 2006; 11(2):110-9.

16. Abegg C. Hábitos de higiene bucal de adultos portoalegrenses. Rev Saúde Pública. 1997; 31(6):586-93.

17. Figueira TR, Leite ICG. Percepções, conhecimentos e práticas em saúde bucal de escolares. RGO. 2009; 56(1):27-32.

18. Fernandes IB, Sousa PFC, Corrêa-Faria P, Ramos-Jorge ML, Marques LS, Corrêa-Faria P. Hábitos parafuncionais em crianças de 36 a 71 meses de idade: prevalência e fatores associados. Arq Cent Estud Curso Odontol Univ Fed Minas. 2013; 49(3):126-32.

19. Nóbrega MTC, Freire JCP, Dias-Ribeiro E, Ghersel H, Ghersel ELA. Avaliação da percepção de gestantes sobre as doenças cárie e periodontal. Arch. Health Invest, 2016; 5(5):247-50.

20. Gonçalves PE, Oliveira YS, Seixas FL. Educação em saúde bucal por meio de instrumentos de higiene oral. FOL-Faculdade de Odontologia de Lins/Unimep . 2013; 23(2):35-44.

21. Carvalho VLR, Mesas AE, Andrade SM.. Aplicação e análise de uma atividade de educação em saúde bucal para idosos. Espaç Saúde, 2006; 7(2):1-7.

\section{CONFLITO DE INTERESSES}

Os autores declaram não haver conflitos de interesse.

\section{AUTOR PARA CORRESPONDÊNCIA}

José Klidenberg de Oliveira Júnior

joseklidemberg@gmail.com

Submetido em 05/12/2016

Aceito em 06/01/2017 